\title{
COMPETENCIAS EN LECTURA E INTERPRETACIÓN DE GRÁFICOS ESTADÍSTICOS DE LA PRENSA EN FUTUROS DOCENTES DE MATEMÁTICAS EN ITALIA Y PORTUGAL
}

\section{SKILLS IN READING AND INTERPRETING STATISTICAL GRAPHS FROM PRESS IN FUTURE MATH TEACHERS IN ITALY AND PORTUGAL}

\author{
José A. Garzón-Guerrero \\ Universidad de Granada \\ jgarzon@ugr.es \\ Rocío Álvarez-Arroyo \\ Universidad de Granada \\ rocioaarroyo@ugr.es
}

\section{Resumen}

Gran parte de la masiva información de los medios de comunicación actuales viene dada en forma de datos y gráficos estadísticos, que ayudan a dar credibilidad y a identificar más rápidamente la información relevante. Es fundamental entonces, que los individuos, especialmente los futuros profesionales de la enseñanza, sean capaces de leer e interpretar dichos diagramas de forma eficaz y crítica. En este trabajo se han analizado las competencias gráficas en estadística de futuros formadores de Italia y Portugal, mediante el análisis de dos tipos de gráficos muy usados en la prensa: los gráficos de líneas y los pictogramas. Seleccionando gráficos que contenían errores de construcción, se planteó a los participantes un cuestionario acerca de su comprensión e interpretación de los mismos. En los resultados del estudio aparecen carencias en la detección de errores y en la modificación de gráficos. Se obtuvieron mejores resultados en los gráficos de líneas que en los pictogramas. En general, se observa una insuficiente formación estadística que dificulta la correcta lectura y la interpretación crítica de los gráficos estadísticos.

Palabras-clave: gráficos estadísticos, gráfico de líneas, pictograma, errores y dificultades, cultura estadística, futuros profesores.

\begin{abstract}
Nowadays, most of the massive information from the media comes in the form of statistical data and graphs, which help to provide credibility and identify relevant information more quickly. Therefore, it's essential that citizens, especially future teachers, are able to read and interpret such charts effectively and critically. In this paper we analyze the graphic skills of prospective teachers in Italy and Portugal, with two types of graphics widely used in the press: line charts and pictograms. By selecting graphics with construction errors, the participants were asked to answer a questionnaire about their understanding and interpretation. In the results, there were shortcomings in skills involving detection of errors and modification of graphs. The best results were obtained for line charts than for pictograms. In general, there is insufficient statistical training, making the correct reading and critical interpretation of graphics harder.
\end{abstract}


Keywords: statistical graphs, line graphs, pictograms, errors and difficulties, statistical literacy, future teachers.

\section{INTRODUCCIÓN}

La necesidad de la sociedad actual de consumir información de manera inmediata y en casi cualquier lugar obliga a que los medios de comunicación cuenten con las herramientas adecuadas para poder ejecutar con fiabilidad ese proceso. Tanto desde los medios denominados tradicionales (prensa escrita, radio, televisión...) como desde los digitales (sitios web, redes sociales...), se crea un flujo informativo masivo y constante que llega a todos los ciudadanos, que son los que finalmente deben seleccionar lo que les interesa y les sirva para tomar decisiones. Para ayudar a los lectores, y también para aportar rigor y verosimilitud, gran parte de las noticias que aparecen en los medios incluyen información en forma de resultados estadísticos, en principio más sencillos de entender. Pero en gran parte de las ocasiones este proceso no siempre se realiza de la forma más adecuada, puesto que existen carencias en la formación matemática y estadística de lector.

Es fundamental que los ciudadanos posean las herramientas y los conocimientos estadísticos necesarios para poder actuar con solvencia, tener una actitud crítica ante esos datos para llegar a una correcta interpretación y ser capaces de detectar posibles sesgos (BATANERO et al., 2014; ENGEL, 2019). Todas estas habilidades están incluidas dentro de la denominación de cultura o alfabetización estadística (statistical literacy, en inglés). Para un individuo, poseer cultura estadística supone tener conocimientos, destrezas, razonamientos, intuiciones y actitudes sobre la estadística que le ayuden a entender y evaluar críticamente los resultados estadísticos que aparecen en la vida cotidiana (BATANERO, 2004). Los conocimientos y destrezas se refieren a los conceptos y habilidades estadísticas básicas que debe tener el ciudadano. El razonamiento incluye todas las capacidades de pensamiento fundamental estadístico. Las intuiciones sobre el azar deben ser controladas y diferenciar las que son correctas usando el razonamiento a la hora de tomar decisiones. Las actitudes comprenden todos los sentimientos y emociones que experimenta y que, si son negativas, interfieren a la hora de alcanzar correctamente los objetivos, al igual que la valoración de la estadística como instrumento (BATANERO, 2004).

En este aspecto, los profesores ejercen un papel fundamental para la adquisición de 
las capacidades gráficas en los estudiantes que serán los ciudadanos del porvenir, ya que sobre los profesores recae la tarea de que los alumnos asimilen los conceptos estadísticos elementales desde los primeros niveles educativos (BATANERO et al., 2014; ZIEFFLER; GARFIELD; FRY, 2018). Se hace necesaria, por tanto, la existencia de una sólida formación estadística del profesorado, y por ello es conveniente evaluar su nivel inicial de cultura estadística para, posteriormente, actuar de manera consecuente estableciendo las acciones pedagógicas que sean convenientes (GONZÁLEZ; ESPINEL; AINLEY, 2011).

Dentro de los contenidos en estadística, la lectura e interpretación de gráficos elementales es una de las habilidades básicas que una persona estadísticamente culta debe poseer. Los gráficos estadísticos suelen ser el tipo de representación más utilizado en prensa, ya que permite evaluar la información más rápidamente y de manera visual (RUZ et al., 2018) y además son autoexplicativos, esto es, contienen en sí mismos la suficiente información para comprender la noticia sin necesidad de leer todo el texto (SHARMA, 2013). Pero el individuo con una adecuada cultura estadística no puede limitarse a una lectura literal de cada una de las partes del gráfico, sino que debe usar su formación para interpretar todos los aspectos presentes en él, que le sirvan para comunicar al resto de individuos las opiniones y conclusiones fundamentadas en la información extraída cuando sea relevante (GAL; MURRAY, 2011).

El uso de gráficos provenientes de la prensa para la enseñanza de la estadística a través de actividades pedagógicas ha sido usado con anterioridad, ya que pueden ser una forma atractiva de proporcionar una motivación inicial a los alumnos y además fijar las bases para establecer las etapas de aprendizaje sobre el tema (MONTEIRO; AINLEY, 2010).

En este trabajo se han evaluado las competencias en lectura e interpretación crítica usando dos de los tipos de gráficos estadísticos básicos más utilizados en la prensa (de líneas y pictogramas) en futuros docentes de Educación Primaria y Secundaria, de Italia y Portugal, comparando los resultados con estudios similares realizados en España y en otros países, de donde podría derivarse la creación de actividades formativas acerca de la comprensión gráfica (GEA; ARTEAGA; CAÑADAS, 2017) que ayuden a mejorar la cultura estadística de los docentes. 


\section{MARCO TEÓRICO}

La enseñanza de los gráficos, y por consiguiente de la estadística, está presente dentro de las asignaturas de matemáticas en la mayoría de los currículos educativos de casi todos los países del mundo, desde los niveles más bajos hasta los universitarios (ARTEAGA et al., 2016; BATANERO et al., 2014; WU; WONG, 2009). Pero la lectura de gráficos que se realiza en estos niveles suele ser una tarea bastante específica, al contrario de las necesidades que pueden aparecer en el mundo real. Esta contradicción puede incidir en que no se puedan formar individuos competentes en ese sentido (ESPINEL, 2007). En las aulas, el trabajo con los gráficos se centra de forma casi exclusiva en hallar las respuestas matemáticamente correctas, y que a menudo vienen descontextualizadas, mientras que fuera del ámbito escolar es siempre necesario conocer tanto el contexto como el contenido (MONTEIRO; AINLEY, 2010; WATSON; FITZALLEN, 2010).

El orden en que los diferentes tipos de gráficos van siendo introducidos en las diferentes etapas escolares mantiene una estructura común en casi todo el mundo. En las fases de Infantil o primeros cursos de Primaria se comienza con la lectura e interpretación de gráficos muy simples, pictogramas o gráficos de barras sencillos, para familiarizar a los alumnos con el tema. Por ejemplo, suelen construirse diagramas de barras de forma manipulativa mediante palos o dibujos que, siendo convenientemente apilados o adosados, forman la altura de cada una de las barras (ALSINA; VÁSQUEZ; MUÑIZ-RODRÍGUEZ; RODRÍGUEZ-MUÑIZ, 2020). A lo largo de la Primaria se trabaja sobre todo con gráficos de barras y se introducen los gráficos de líneas. Los gráficos de sectores o circulares aparecen en últimos cursos de la Primaria o incluso en la Educación Secundaria, en cuya etapa se pretende dominar la interpretación y construcción de todas las clases de dichos diagramas. Esto se puede comprobar con la semejanza en los currículos actuales europeos y de otros países (por ejemplo, en España, Batanero et al., 2014; en Portugal, Ponte; Fonseca, 2001; en Italia, Ottaviani; Luchini, 2005; en Singapur, Wu; Wong, 2009). Otros gráficos ni siquiera aparecen en el currículum de ninguna etapa educativa, como mapas coropléticos u otros no convencionales, pero que son usados a menudo en los medios de comunicación (ALSINA; VÁSQUEZ; MUÑIZ-RODRÍGUEZ; RODRÍGUEZ-MUÑIZ, 2020). 


\section{Competencias en lectura e interpretación de gráficos estadísticos}

Los gráficos estadísticos exigen al lector varios procesos de interpretación, comenzando por el análisis de elementos estructurales del gráfico (títulos, ejes, etiquetas...), pasando por la percepción de las variables y escalas que aparecen (si el origen comienza en cero, rango de valores...) y finalizando con las conclusiones sobre los niveles de cada variable y su relación con la realidad que se busca representar (percibir tendencias, relación entre variables...; en Arteaga et al., 2011a), son, por tanto, objetos semióticamente complejos (BATANERO; ARTEAGA; RUIZ, 2010). Añadiendo cierta dificultad al proceso cognitivo, cada clase de gráfico posee sus propios convenios de interpretación. Esa dificultad semiótica puede provenir de la limitación de la comprensión del lenguaje (gráfico, verbal, numérico...), de los procedimientos (recuento de datos, cálculo de frecuencias...) o de los conceptos y proposiciones, como la proporcionalidad, la distribución de la variable...(PALLAUTA; ARTEAGA, 2020). La comprensión gráfica implica que el lector tenga unas ciertas habilidades que le permitan entender el significado del mismo. Diferentes habilidades provocan diferentes niveles de comprensión (FRIEL; CURCIO; BRIGHT, 2001). Así, alcanzar el completo desarrollo de la comprensión de un gráfico es un proceso gradual, que necesita de un aporte de gran variedad de gráficos y de problemas asociados a ellos (GONZÁLEZ; ESPINEL; AINLEY, 2011).

En la literatura podemos encontrar varios modelos teóricos que establecen diferentes clasificaciones jerárquicas en los niveles de lectura y comprensión de gráficos, desde la incapacidad de extraer ningún dato de los diagramas hasta la habilidad de predecir y extrapolar datos que no se encuentran visibles explícitamente. En Wu y Wong (2009) se considera un marco que tiene en cuenta cuatro aspectos en la comprensión de gráficos estadísticos: Lectura de Gráficos, en el que se sabe extraer los datos directamente o comparar parte de ellos; Interpretación de los Gráficos, cuando se forman opiniones y se realizan inferencias acerca de los datos; Construcción de Gráficos, el individuo es capaz de reflejar datos en de forma gráfica; Evaluación de Gráficos, tener la capacidad de reconocer si un gráfico es correcto y efectivo.

Para alcanzar el nivel más alto es necesario que el lector adquiera unas competencias fundamentales para la comprensión del lenguaje gráfico. En Friel, Curcio y Bright (2001) se exponen seis habilidades o destrezas relacionadas que deben de poseer los 
consumidores de gráficos estadísticos, que aquí se resumen en cuatro componentes:

- Reconocer las componentes estructurales del gráfico y sus relaciones: distinguir cada uno de estos elementos individualmente (escala, ejes, etiquetas...), interpretarlos y ser capaz de distinguir si son apropiados para la inclusión en el gráfico.

- Percibir el impacto de dichos elementos sobre la presentación de la información: ser capaz de, por ejemplo, predecir el cambio del gráfico si cambiase la escala, o percatarse de si la escala usada es incorrecta por no ser proporcional. En particular, saber detectar que un gráfico puede estar sesgado por la variación, intencionada o no, de un elemento estructural del mismo.

- Trasladar las relaciones que aparecen en el gráfico a los datos que se representan en el mismo y viceversa: por ejemplo, relacionar las variables entre sí, o saber que una gráfica debe ser creciente cuando la relación entre dos variables es directa. Interpretar la información que se presenta en el gráfico.

- Reconocer si un gráfico es más adecuado que otro: saber elegir el tipo de gráfico más útil teniendo en cuenta el tipo de variable que se está representando y el tipo de problema que se quiere abordar.

Estas competencias se corresponden a los diferentes comportamientos que aparecen entre los estudiantes a la hora de entender un gráfico y que dan cuenta del llamado Sentido Gráfico (SHARMA, 2013): leer, describir, interpretar, analizar, predecir y extrapolar datos. En este trabajo se utilizará el modelo de competencias de Friel, Curcio y Bright (2001) descrito anteriormente para estudiar la comprensión gráfica en futuros docentes.

Uno de los marcos teóricos más utilizados en investigación de gráficos es el propuesto por Shaughnessy, Gardfield y Greer (1996) y Friel, Curcio y Bright (2001) con cuatro etapas en la lectura: lectura de los datos (es capaz de extraer datos directamente del gráfico); lectura entre los datos (puede encontrar relaciones entre los datos); lectura más allá de los datos (puede extrapolar y realizar predicciones); lectura detrás de los datos (realiza una lectura crítica y se plantea la fiabilidad del gráfico).

Aoyama (2007) describe cinco niveles para la lectura de los datos basados en lo que el lector es capaz o no de hacer, con cada nivel posterior conteniendo a todos los anteriores: nivel idiosincrático (no es capaz de leer nada correctamente); nivel de lectura básico 
(puede leer datos y ver tendencias); nivel literal (puede describir significados del contexto del gráfico, pero sólo literalmente); nivel crítico (comprende el contexto y analiza la fiabilidad de la información); nivel de hipótesis (puede aportar sus propias hipótesis o modelos).

\section{Estudio sobre lectura de gráficos}

Como se ha comentado, uno de los sectores de la población donde es más importante la necesidad de poseer una adecuada cultura estadística, incluyendo los gráficos, son los profesores de Educación Primaria o Secundaria o los que algún día llegarán a serlo, ya que de ellos depende que el resto de los ciudadanos adquieran las competencias básicas que les ayuden a desenvolverse adecuadamente con la estadística (ARTEAGA et al., 2011b).

Una búsqueda bibliográfica ofrece varios resultados de trabajos de investigación que abordan esta temática (GONZÁLEZ; ESPINEL; AINLEY, 2011). En Monteiro y Ainley (2007) se estudia la lectura de gráficos de la prensa diaria en futuros profesores de primaria de Brasil e Inglaterra, y se llega a la conclusión de que la mayoría de ellos no poseían suficiente conocimiento matemático para poder ni siquiera leerlos y que muchos de los que sí lo hacían no eran capaces después de interpretarlos correctamente o de hacer una lectura crítica. Espinel (2007) estudia la interpretación de gráficos en futuros profesores de primaria españoles en comparación con estudiantes norteamericanos de distintos niveles educativos. Las conclusiones indican que los futuros profesores tienen peor rendimiento que el de los encuestados estadounidenses.

En un amplio estudio de más de un millar de participantes, no exclusivamente profesores, Schonlau y Peters (2008) muestran que el tiempo de observación de un gráfico por un individuo influye en una interpretación más precisa del mismo. Arteaga et al. (2016) estudia la construcción de gráficos elementales en más de doscientos futuros profesores de primaria, en los que aparecen conflictos semióticos relacionados con convenios de construcción, sentido numérico y otros. Molina-Portillo et al. (2018) y Ruz et al., (2018), se centran en la interpretación crítica de datos en futuros profesores de primaria a través de gráficos de barras publicados en medios de comunicación y concluyen que existen bastantes déficits en la formación de los estudiantes. Los gráficos de líneas han sido usados en Peebles y Ali (2009), donde se compara el rendimiento entre este tipo de diagramas y 
los de barras cuando hay involucrados datos de tres variables, considerándose que los estudiantes cometen menos errores cuando se usan los gráficos de barras. En Berg y Smith (1994) se utilizan los gráficos de líneas para comprobar que, aunque las carencias en la lectura son altas en general, el tipo de preguntas de los cuestionarios usados influye en el resultado final y es más conveniente usar pregunta de respuesta libre. La mayor parte de los estudios existentes coinciden en que, en general, a pesar de la importancia que tiene la interpretación de la información estadística a través de gráficos, se observan bastantes dificultades y errores en dicha interpretación (PÉREZ-ECHEVERRÍA; POSTIGO; MARÍN, 2018) y pueden existir sesgos, intencionados o no, que la compliquen aún más (ARTEAGA et al., 2016).

\section{METODOLOGÍA}

Para cumplir los objetivos de este estudio, los autores se han desplazado a dos países pertenecientes a la Unión Europea para seleccionar una muestra de 52 futuros docentes de Educación Primaria y Secundaria, cursando sus estudios 29 de ellos en Portugal (de la Universidade de Tras-os-Montes e Alto Douro, de la localidad de Vila-Real) y 23 en Italia (de la Universitá Roma III, Roma). La mayor parte de los participantes eran alumnos que realizaban el último curso de estudios de grado y lo que en España equivaldría a un máster de formación del profesorado. El resto estaban integrados en los primeros años de un doctorado en Educación. Al grupo de participantes italianos se unieron tres profesores noveles en activo, con no más de dos años de experiencia. En ambos países, la estadística y los gráficos son parte del currículum básico de educación desde los primeros niveles, como se ha visto en la sección anterior. En el máster y en el doctorado también se impartían asignaturas que tenían estadística como parte de su temario. Los datos se recogieron durante estancias de trabajo en dichas universidades, en las que uno de los autores impartió talleres de estadística a los docentes y estudiantes colaboradores.

A los participantes se les presentó un cuestionario, validado de forma interna, compuesto por gráficos extraídos de la prensa escrita junto con algunas cuestiones que debían de responder al inicio de las sesiones. Una vez recogidos los datos, se debatía con ellos sobre la competencia gráfica, analizando el cuestionario que acaban de rellenar, para de este modo aumentar su conocimiento matemático y didáctico en el tema. Se eligieron 
cuatro tipos de gráficos diferentes: de sectores, de barras, de líneas y pictogramas, ya que
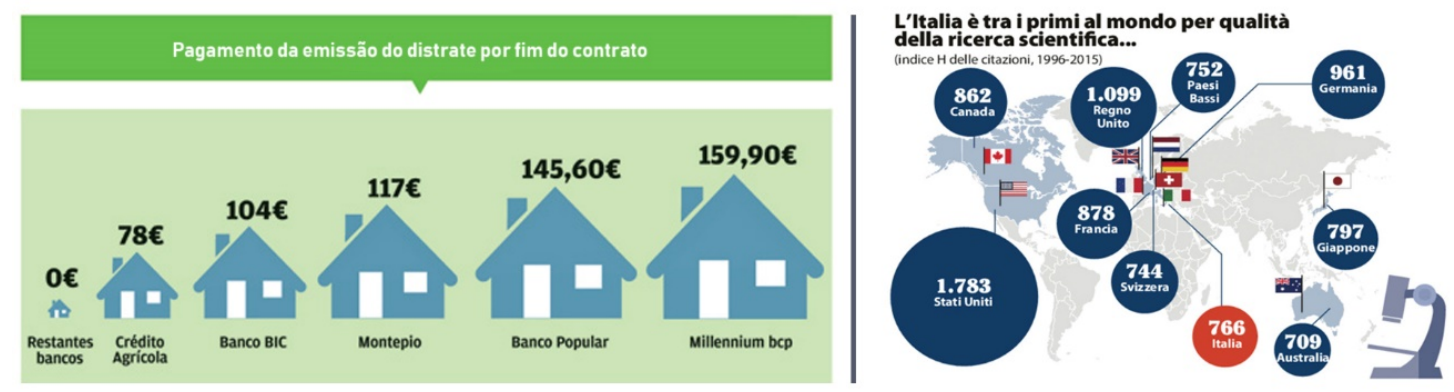

suelen ser los más habituales en la prensa.

La elección de los gráficos no se hizo de manera arbitraria. Cada uno de los tipos de gráfico fue escogido de forma específica entre las publicaciones de cada país en fechas recientes, lo que requirió un exhaustivo trabajo previo de búsqueda en la prensa interna de cada estado participante. Se efectuó una investigación entre los medios de comunicación escritos de ambos países, buscando gráficos adecuados para su estudio, intentando localizar aquellos que tuvieran algún tipo de error o sesgo en su construcción para probar las competencias presentes en la lectura e interpretación de los diagramas. En este informe sólo se analizarán los resultados derivados de los tipos de gráfico de líneas y pictogramas. Los otros dos han sido descritos en un análisis exploratorio previo (GARZÓNGUERRERO; JIMÉNEZ CASTRO, 2021).

En la Figura 1 se observan los casos que han sido seleccionados para la clase de diagrama de líneas y en la Figura 2 para los pictogramas, todos ellos extraídos de la prensa italiana y portuguesa.

Figura 1 - Gráficos de líneas para la prueba en Portugal (izquierda) e Italia (derecha).

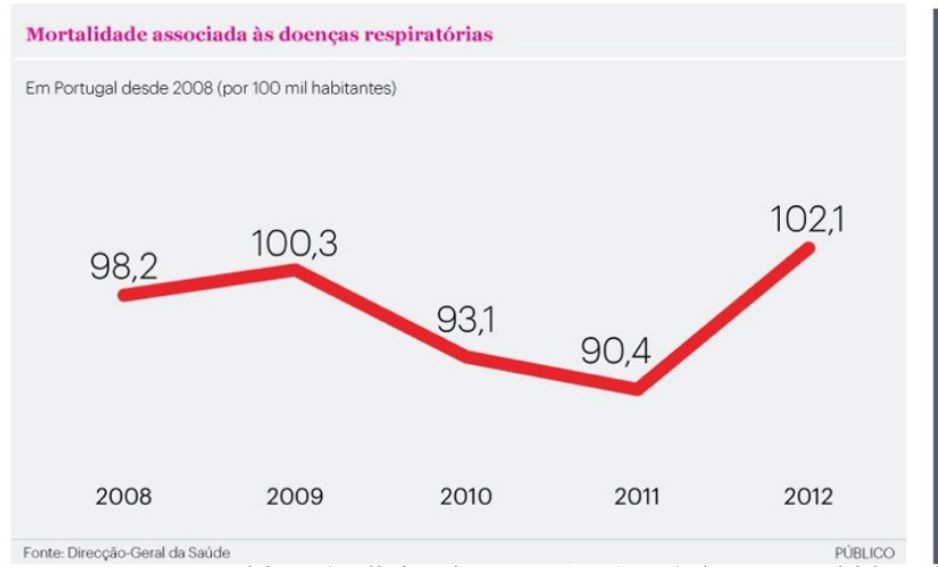

Le vaccinazioni

Copertura vaccinale in Italia valori in \%

- VACCINAZIONI OBBLIGATORIE - VACCINAZIONI CONSIGLIATE

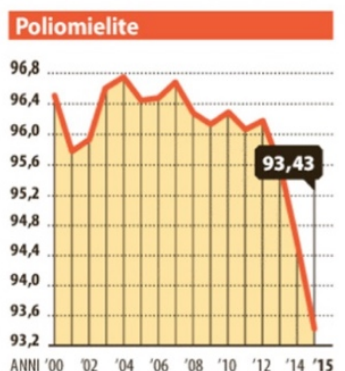

Fuente: Publico (9 diciembre 2014, pág. 5) / La Repubblica (12 mayo 2017, pág. 6).

Figura 2 - Pictogramas usados para la prueba en Portugal (izquierda) e Italia (derecha). 
Fuente: Jornal de Negócios (2 junio 2014, pág. 17) / La Repubblica (19 enero 2017, pág. 24).

Se construyeron cuatro preguntas relacionadas con las cuatro competencias gráficas adaptadas de Friel et al. (2001) descritas en la sección anterior y traducidas a la lengua oficial del lugar dónde tenía lugar la experiencia. Los cuestionarios fueron distribuidos a los participantes, que debían de responder a esas cuatro cuestiones para cada una de las imágenes correspondientes a cada tipo de gráfico:

- P1. Descripción del gráfico: describir el tipo de gráfico, variables, criterio para mostrar la información...

- P2. ¿Puede encontrar algún sesgo o error en el gráfico?

- P3. ¿Qué información relevante puede extraer del gráfico?

- P4. ¿Confía en el medio de comunicación del gráfico? ¿Considera que es el mejor tipo de gráfico para explicar la información? Aporte otro para mejorarlo.

El cuestionario se les entregaba a principio la primera sesión, sin más información ni contexto que la propiamente contenida en el gráfico. Tras su realización se impartió a los asistentes un seminario específico sobre el tema donde se consideraron los ejemplos sobre los que antes habían tenido la oportunidad de contestar, haciéndoles entrega también de la página completa del periódico o revista de la noticia, teniendo así toda la información que había disponible.

Se ha llevado a cabo un análisis cualitativo, de carácter exploratorio, para evaluar las respuestas de los cuestionarios obtenidos y se ha realizado un análisis descriptivo de las contestaciones a cada pregunta formulada. Los resultados han sido clasificados en dos categorías principales: incorrecta o correcta. Se agrupan en incorrectas cuando el alumno no contesta a la pregunta o si todo lo que ha escrito es erróneo. Se consideran correctas cuando los encuestados contestan a todas las preguntas de forma correcta o parcialmente correcta y que contiene afirmaciones válidas pero incompletas.

\section{Análisis de los gráficos del cuestionario}

Antes de realizar el análisis cualitativo es necesario describir los gráficos seleccionados teniendo como referencia las cuatro cuestiones planteadas, y establecer las respuestas correctas, de modo que se pueda hacer la categorización de los resultados de forma más efectiva y confiable. 
Todo lo relativo a descripción del gráfico e identificación de sus componentes corresponde a la primera cuestión planteada. Para la Figura 1 ambas representaciones corresponden a un gráfico de líneas simple, aunque en el caso de la imagen italiana parece también un diagrama de área por la existencia de una zona sombreada. Este tipo de gráficos suele usarse para presentar secuencias temporales y muestra, generalmente sobre unos ejes cartesianos, la relación que existe entre dos o más variables, representando cada una en un eje. En el caso portugués (izquierda) no se muestran explícitamente los ejes, aunque sí las marcas en abscisas de la variable tiempo. Las variables que aparecen son la mortalidad asociada a enfermedades respiratorias (en número de personas por cada 100000 habitantes) y la cobertura de vacunación infantil (en porcentaje), para Portugal e Italia, respectivamente. La variable que relaciona a ambas es temporal (en años).

De la Figura 2, se extrae que ambas imágenes son pictogramas bidimensionales de área o tamaño. Entre los pictogramas, suelen encontrarse dos tipos: numérico (o también llamado de barras, formado por figuras de igual tamaño que se repiten, cuyo número es proporcional a la frecuencia representada) o de tamaño, como en nuestro caso (o de escala, en el que las áreas de cada figura representan la frecuencia de la variable (WU; WONG, 2009). La variable del caso portugués (izquierda) es el precio de la comisión de rescisión del contrato hipotecario (en euros) en diferentes entidades bancarias. En el caso italiano (derecha) las variables son el índice $\mathrm{H}$ de los investigadores (relacionado con número de citas de artículos de investigación) desde 1996 a 2015 en diferentes países del mundo. La forma de representación usa variaciones en el área de un dibujo de una casa (izquierda) o mediante diferentes tamaños de círculos (derecha)

Existen sesgos o errores de construcción en todos los gráficos presentados en este documento, ya que en el proceso de selección de los mismos se marcó esa característica como necesaria. En la Figura 1 (izquierda) no se dibuja ninguno de los ejes, sobre todo se hace evidente con el de ordenadas, que no tienen marcas de numeración, por lo que desconocemos la escala. En ambas imágenes de la Figura 1, el eje de ordenadas no comienza en cero, con lo que la percepción visual de la forma de los diagramas puede resultar engañosa. Restringiendo demasiado los valores en el eje $Y$ tiende a hacer que el hecho que se muestra parezca más exagerado de lo puede reflejar la realidad de los datos. En el caso de la figura italiana (derecha) se pueden observar más errores. En primer lugar, 
los intervalos de tiempo deberían de ser del mismo tamaño, vemos que cada marca se realiza cada dos años menos la última, que lo hace en uno sólo. Los valores en la escala de ordenadas tampoco están correctamente elegidos, ya que hay rangos que es mejor evitar (WONG, 2010). Otro error es el de sombrear con otro color el área bajo la curva, ya que lo convierte en un gráfico de área, pero no de forma apropiada, ya que la gráfica no comienza en cero y además el color puede confundirse con la leyenda de otro gráfico que está colocado en la parte superior. Por tanto, esa elección en el diseño no da ninguna información relevante.

En ambos pictogramas de tamaño (Figura 2) también hay errores de construcción, puesto que en este tipo de gráficos es necesario que el valor de la variable sea proporcional al tamaño o área de la figura, cosa que no ocurre en ninguno de los dos casos y que puede llevar a confusión. Es un error que aparece de manera típica en esta clase de diagramas, principalmente en la prensa, cosa que no ocurre en los pictogramas numéricos (WU; WONG, 2009). En la Figura 2 (izquierda) han elegido la altura de la casa, y no el área, como indicador de la variable y en la de la derecha se han construido los círculos atendiendo a la proporcionalidad de su diámetro con la variable, y no su tamaño.

En referencia a la tercera pregunta, hay que decir que el gráfico se entregó a los participantes descontextualizado y sólo tras el test se les mostró la noticia completa. En la Figura 1 (izquierda) se puede observar que la mortalidad debido a enfermedades respiratorias creció en 2012 después de haber bajado en dos años consecutivos, aunque en principio la proporción de subida no es muy exagerada. En el gráfico italiano (Figura 1, derecha), se presenta el porcentaje de la población infantil que está vacunada contra la poliomielitis. Por la línea color se comprueba que desde el año 2000 al 2012, la tasa de vacunados ha variado entre un rango de un 1\% y que a partir del 2012 ha caído casi tres puntos en los tres últimos años. De la Figura 2 (izquierda) puede extraerse que dependiendo del banco en el que se encuentre la hipoteca, cancelarla puede costar desde cero euros hasta casi 160, con lo cual si no queremos pagar dicha comisión lo ideal sería evitar esas entidades. En la Figura 2 (derecha) vemos que tras Estados Unidos (la mayor, aunque exagerada) hay una segunda línea de países punteros en cuanto a publicaciones y citas científicas (Reino Unido, Alemania...) y que Italia les sigue de cerca a no mucha distancia.

En cuanto a la última cuestión, todos los gráficos podrían mejorarse evitando los 
errores y sesgos mencionados. Para los diagramas de líneas podría utilizarse como representación alternativa gráficos de barras. Por ejemplo, para la Figura 1 (izquierda) un diagrama de barras que muestre las diferencias. Para el pictograma de la izquierda (Figura 2) debería usarse un gráfico de barras o simplemente una tabla ordenada con todos los valores. Para el caso italiano de la Figura 2 (derecha) también podría usarse un diagrama de barras, una tabla, o un gráfico de sectores disminuyendo el número de categorías.

\section{RESULTADOS Y DISCUSIÓN}

Para este trabajo sólo se han considerado las respuestas a los casos del gráfico de líneas y de los pictogramas. Una vez recogidos los cuestionarios, se llevó a cabo un análisis de su contenido para comprobar la corrección de la respuesta, comparar entre preguntas, gráficos y países.

Los resultados de las respuestas consideradas como correctas para las descripciones del gráfico de líneas se muestran la Tabla 1 . Se ha de mencionar de nuevo que las preguntas no respondidas se han considerado como incorrectas.

Como se puede observar, la gran mayoría de los evaluados $(72,4 \%$ y $65,2 \%$ en Portugal e Italia, respectivamente) pudieron describir correctamente el tipo de gráfico, variable, escalas y parámetro de representación. Esto puede indicar que el gráfico de líneas es bien conocido por los participantes, incluso en los casos de construcciones y formatos no estándar, cómo los propuestos. También la mayoría, aunque en menor proporción (un $65,5 \%$ y 69,6\%), fueron capaces de extraer información útil de los mismos, es decir, pudieron situar los datos en un contexto e identificar cuáles eran los datos fundamentales, aunque no se mostraran explícitamente.

Tabla 1 - Descripciones correctas para el gráfico de líneas (\% respecto a la muestra) para los dos países.

\begin{tabular}{lcccc}
\hline \multicolumn{1}{c}{ Líneas } & P1 (\%) & P2 (\%) & P3 (\%) & P4 (\%) \\
\hline Portugal & 72,4 & 27,6 & 65,5 & 17,2 \\
Italia & 65,2 & 34,8 & 69,6 & 26,1 \\
\hline Total & $\mathbf{6 9 , 2}$ & $\mathbf{3 0 , 8}$ & $\mathbf{6 7 , 3}$ & $\mathbf{2 1 , 2}$ \\
\hline \multicolumn{5}{c}{ Fuente: elaboración propia. }
\end{tabular}

Muchos menos participantes fueron capaces de detectar errores o sesgos, solamente 
el $27,6 \%$ y el $34,8 \%$ de los encuestados. Esto podría implicar un razonamiento proporcional incompleto en los participantes, que coincide con lo mostrado por Arteaga et al. (2011b) en futuros profesores en España al construir sus gráficos, ya que muchos de ellos erraban al usar escalas no proporcionales. También es escasa la cantidad de personas $(17,2 \%$ y 26,1\%) que cambiaría el gráfico por una representación más adecuada.

Atendiendo a los pictogramas, el porcentaje de respuestas correctas se muestra en la Tabla 2.

Tabla 2 - Descripciones correctas para los pictogramas (\% respecto a la muestra) para los dos países.

\begin{tabular}{lcccc}
\hline Pictogramas & P1 (\%) & P2 (\%) & P3 (\%) & P4 (\%) \\
\hline Portugal & 75,9 & 6,9 & 55,2 & 27,6 \\
Italia & 73,9 & 17,4 & 47,8 & 30,4 \\
\hline Total & $\mathbf{7 5 , 0}$ & $\mathbf{1 1 , 5}$ & $\mathbf{5 1 , 9}$ & $\mathbf{2 8 , 8}$ \\
\hline
\end{tabular}

Fuente: elaboración propia.

Los resultados son similares a los obtenidos para los de líneas en cuanto a la lectura directa de los datos del gráfico ( $\mathrm{P} 1,75,9 \%$ y 73,9\%) pero algo más baja en cuanto a la extracción de tendencias, contextos e interpretación (P3, 55,2\% y 47,8\%). Sin embargo, es llamativo que en esta clase de diagramas el número de personas que fue capaz de detectar errores disminuyó enormemente $(6,9 \%$ y $17,4 \%)$ pero como contrapartida, hubo más participantes que supieron mejorar y aportaron un cambio correcto en el tipo de gráfico $(27,6 \%$ y $30,4 \%)$. Esto puede ser debido a que, si bien los pictogramas son mostrados en los primeros años de enseñanza de la estadística, lo son los de tipo numérico y no los de tamaño o área, que en particular no están muy trabajados, y también porque la percepción de la proporcionalidad es más fácil con relaciones lineales que cuadráticas.

Otro de nuestros objetivos era saber si existen diferencias en la interpretación y lectura de los estudiantes según el tipo de gráfico. El porcentaje de respuestas correctas según la clase de diagrama se puede encontrar en la Tabla 3, para cada una de las preguntas. En todos los casos, las preguntas 1 y 3 son las que mejores resultados obtienen, seguidas de la cuarta y la segunda. Las medidas son parecidas, pero se observa que, en general, existe un mejor desempeño a la hora de interpretar los gráficos de barras $(48,6 \%)$ y de líneas $(47,1 \%)$ que los de sectores $(42,8 \%)$ o los pictogramas (41,8\%). Esto puede deberse a que los gráficos de barras y de líneas son los que más se trabajan en el currículo de estos 
países, España incluida, desde los primeros cursos de Primaria, mientras que el diagrama de sectores sólo se comienza a estudiar en el último o penúltimo curso de la secundaria. Aunque los pictogramas son introducidos a edades muy tempranas, incluso en infantil, no son del tipo elegido en este trabajo, de tamaño o área, sino que son más simples, numéricos o de barras. Así, con esta categoría de diagramas no suelen ser tratados en la escuela (WU; WONG, 2009).

Tabla 3 - Respuestas clasificadas como correctas para las cuatro cuestiones para cada tipo de gráfico y para el total (\% respecto a la muestra).

\begin{tabular}{lccccc}
\hline \multicolumn{1}{c}{ Tipo de gráfico } & P1 (\%) & P2 (\%) & P3 (\%) & P4 (\%) & Total \\
\hline Gráfico de líneas & 69,2 & 30,8 & 67,3 & 21,2 & $\mathbf{4 7 , 1 \%}$ \\
Pictogramas & 75,0 & 11,5 & 51,9 & 28,8 & $\mathbf{4 1 , 8 \%}$ \\
Gráfico de barras & 80,8 & 23,1 & 71,2 & 19,2 & $\mathbf{4 8 , 6 \%}$ \\
Gráfico de sectores & 71,2 & 21,2 & 65,4 & 13,5 & $\mathbf{4 2 , 8 \%}$ \\
\hline Total & $\mathbf{7 4 , 1}$ & $\mathbf{2 1 , 7}$ & $\mathbf{6 4 , 0}$ & $\mathbf{2 0 , 7}$ & \\
\hline
\end{tabular}

Fuente: adaptado de Garzón-Guerrero y Jiménez Castro (2021).

En la Figura 3 se presentan los resultados globales por pregunta para cada país, para investigar si en alguna de ellas hay alguna desigualdad entre los dos estados.

Figura 3 - Respuestas correctas (\% respecto a la muestra) para las preguntas realizadas con respecto a los dos tipos de gráficos elegidos para la prueba en Portugal e Italia.

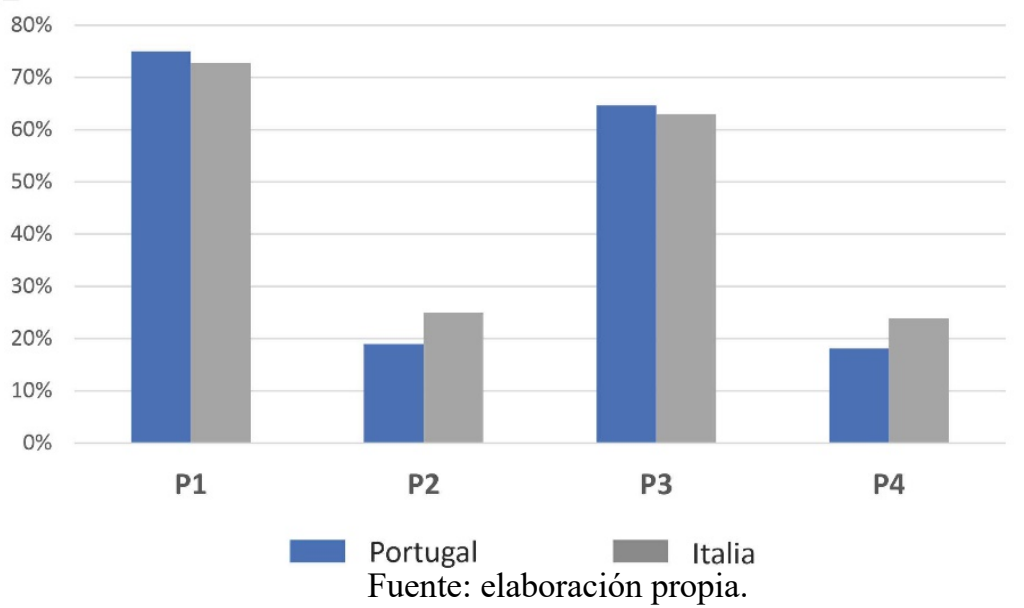

Las diferencias son pequeñas en la gráfica. En una primera aproximación al estudio estadístico, se ha realizado un análisis chi-cuadrado entre los resultados de los dos países para comprobar si las distribuciones eran semejantes. El p-valor resultante era mayor que 0,05 en todos los casos, lo que indica que entre las respuestas de los participantes en ambos países no existen diferencias estadísticamente significativas. Un dato interesante es que 
ninguno de los participantes que respondieron que confiaban en el medio de comunicación del gráfico afirmó que cambiarían el tipo de diagrama, lo que indica una falta de capacidad crítica en la lectura de gráficos (AOYAMA, 2007).

Los porcentajes de éxito obtenidos son semejantes a otros estudios similares (MONTEIRO; AINLEY, 2007; PÉREZ-ECHEVERRÍA; POSTIGO; MARÍN, 2018). En (RUZ et al., 2018) y (MOLINA-PORTILLO et al., 2018) los resultados son un poco más bajos que los de este estudio, debido posiblemente a la inclusión en nuestra muestra de elementos de más alto nivel académico (post-graduados, doctorandos...). También en cuanto a la dificultad de las categorías de gráficos otros estudios muestras conclusiones parecidas, siendo los diagramas de barras y de líneas los que obtienen mejores resultados (PEEBLES; ALI, 2009).

\section{CONCLUSIONES}

En este trabajo se ha realizado un estudio exploratorio de la evaluación de las capacidades de lectura e interpretación de gráficos estadísticos en futuros profesores de matemáticas en Italia y Portugal, usando dos tipos de gráficos fundamentales, de líneas y pictogramas, a través de cuatro preguntas de dificultad variada y realizando diferentes comparaciones. Los resultados han sido altos en cuanto a la diferenciación del tipo de gráfico y a las competencias de identificación de la información representada. Sin embargo, se observan ciertas lagunas en la formación estadística de estos futuros docentes que dificulta la completa interpretación y la lectura crítica de los gráficos de los medios de comunicación. Sobre todo, aparecen ciertas carencias en la detección de sesgos o errores y en la capacidad de corregir o aportar otro tipo de gráficos para mejorar la lectura de la información.

Los resultados obtenidos son semejantes a los que aparecen en trabajos similares realizados entre estudiantes o futuros profesores, en los que muchos participantes no tienen capacidades estadísticas suficientes para realizar una lectura gráfica crítica. Por tanto, es imprescindible, realizar acciones específicas de tipo formativo sobre el profesorado en formación para evitar este tipo de déficits y alcanzar una adecuada cultura estadística. La prensa escrita y otros medios proporcionan una gran gama de posibilidades para poder llevar a cabo esta formación, pues son una fuente inagotable de gráficos como los utilizados 
en este trabajo, que pueden ser usados para motivar y plantear preguntas a los estudiantes que los lleven a mejorar su capacidad de lectura crítica. Además, considerando el avance en la inclusión de temas estadísticos en los currículos educativos desde los primeros cursos, sería conveniente profundizar en la investigación sobre gráficos estadísticos en los libros de texto, ya que constituyen un recurso valioso entre las directrices curriculares y su implementación en el aula que finalmente termina influenciando en el aprendizaje y la cultura estadística del estudiante (HERBEL-EISENMANN, 2007).

\section{Agradecimientos}

Proyecto PID2019-105601GB-I00 / AEI / 10.13039/501100011033, Grupo FQM126 (Junta de Andalucía).

\section{REFERENCIAS}

ALSINA, Á.; VÁSQUEZ, C.; MUÑIZ-RODRÍGUEZ, L.; RODRÍGUEZ-MUÑIZ, L. J. ¿Cómo promover la alfabetización estadística y probabilística en contexto?: estrategias y recursos a partir de la COVID-19 para Educación Primaria. Epsilon: Revista de la Sociedad Andaluza de Educación Matemática "Thales", n. 104, p. 99-128, 2020. AOYAMA, K. Investigating a Hierarchy of Students' Interpretations of Graphs. International Electronic Journal of Mathematics Education, v. 2, n. 3, p. 298-318, 12 dez. 2007.

ARTEAGA, P.; BATANERO, C.; CAÑADAS, G. R.; CONTRERAS, J. M. Las tablas y gráficos estadísticos como objetos culturales. Números. Revista de Didáctica de las Matemáticas, v. 76, p. 55-67, 2011a.

ARTEAGA, P.; BATANERO, C.; ORTIZ, J. J.; CONTRERAS, J. M. Sentido numérico y gráficos estadísticos en la formación de profesores. Publicaciones, v. 41, n. 0, p. 33-49, $2011 b$.

ARTEAGA, P.; BATANERO, C.; CONTRERAS, J. M.; CAÑADAS, G. R. Evaluación de errores en la construcción de gráficos estadísticos elementales por futuros profesores. Revista Latinoamericana de Investigación Matemática Educatica (RELIME), v. 19, n. 1, p. 15-40, 2016.

BATANERO, C. Los Retos de la Cultura Estadística. Yupana, v. 1, n. 1, p. 1-19, 20 nov. 2004.

BATANERO, C.; GEA, M. M.; ARTEAGA, P.; CONTRERAS, J. M. La estadística en la educación obligatoria: análisis del currículo español. Revista Digital Matemática Educación e Internet, v. 14, n. 2, p. 1-14, 2014.

BATANERO, C.; ARTEAGA, P.; RUIZ, B. Análisis de la complejidad semiótica de los gráficos producidos por futuros profesores de educación primaria en una tarea de comparación de dos variables estadísticas. Enseñanza de las Ciencias: Revista de Investigación y Experiencias didácticas, v. 28, n. 1, p. 141-154, 2010. 
BERG, C. A.; SMITH, P. Assessing students' abilities to construct and interpret line graphs: Disparities between multiple-choice and free-response instruments. Science Education, v. 78, n. 6, p. 527-554, 1994.

ENGEL, J. Statistical literacy and society: What is civic statistics? In: III CONGRESO INTERNACIONAL VIRTUAL DE EDUCACIÓN ESTADÍSTICA (CIVEEST) (J. M. Contreras, M. del M. López-Martín, E. Molina-Portillo, Eds.). Actas del Tercer Congreso Internacional Virtual de Educación Estadística. Granada: 2019

ESPINEL, M. C. Construcción y razonamiento de gráficos estadísticos en la formación de profesores. In: XI SIMPOSIO DE SOCIEDAD ESPAÑOLA DE INVESTIGACIÓN EN EDUCACIÓN MATEMÁTICA (M. Camacho, P. Flores, M. del P. Bolea, Eds.) Investigación en educación matemática XI. Tenerife: Sociedad Española de Investigación en Educación Matemática (SEIEM), 2007. Acesso em: 15 set. 2020

FRIEL, S. N.; CURCIO, F. R.; BRIGHT, G. W. Making Sense of Graphs: Critical Factors Influencing Comprehension and Instructional Implications. Journal for Research in Mathematics Education, v. 32, n. 2, p. 124-158, mar. 2001.

GAL, I.; MURRAY, S. T. Responding to diversity in users' statistical literacy and information needs: Institutional and educational implications. Statistical Journal of the IAOS, v. 27, n. 3,4, p. 185-195, 1 jan. 2011.

GARZÓN-GUERRERO, J. A.; JIMÉNEZ CASTRO, M. Estudio de la competencia gráfica de futuros profesores de Portugal e Italia a través de la interpretación de diagramas estadísticos de barras y sectores extraídos de la prensa escrita. Números. Revista de Didáctica de las Matemáticas, v. 106, n. En prensa, p. 33-42, jan. 2021.

GEA, M. M.; ARTEAGA, P.; CAÑADAS, G. Interpretación de gráficos estadísticos por futuros profesores de Educación Secundaria. Avances de Investigación en Educación Matemática, n. 12, p. 19-37, 4 nov. 2017.

GONZÁLEZ, M. T.; ESPINEL, M. C.; AINLEY, J. Teachers' Graphical Competence. In: BATANERO, C.; BURRILL, G.; READING, C. (Eds.). Teaching Statistics in School Mathematics-Challenges for Teaching and Teacher Education: A Joint ICMI/IASE Study: The 18th ICMI Study. New ICMI Study Series. Dordrecht: Springer Netherlands, 2011. p. 187-197.

HERBEL-EISENMANN, B. A. From Intended Curriculum to Written Curriculum: Examining the "Voice" of a Mathematics Textbook. Journal for Research in Mathematics Education, v. 38, n. 4, p. 344-369, 2007.

MOLINA-PORTILLO, E. et al. Evaluación de la interpretación y argumentación estadística de noticias en las que intervienen gráficos de barras en futuros profesores de Educación Primaria. In: XVII CONGRESO DE ENSEÑANZA Y APRENDIZAJE DE LAS MATEMÁTICAS (CEAM XVII) (F. España, Ed.). Actas del XVII Congreso de Enseñanza y Aprendizaje de las Matemáticas: Matemáticas en tierra de cine. Almería: Sociedad Andaluza de Educación Matemáticas Thales, 2018

MONTEIRO, C.; AINLEY, J. Investigating the Interpretation of Media Graphs among Student Teachers. International Electronic Journal of Mathematics Education, v. 2, n. 3, p. 187-207, 12 dez. 2007. 
MONTEIRO, C. E. F.; AINLEY, J. M. The interpretation of graphs: reflecting on contextual aspects. Alexandria: Revista de Educação em Ciência e Tecnologia, v. 3, n. 2, p. 17-30, 1 jul. 2010.

OTTAVIANI, M. G.; LUCHINI, S. R. "Data and Predictions" Emerging as One of the Basic Themes in the Mathematical Curriculum of the First Cycle School Level in Italy. In: (G. Burrill, M. Camden, Eds.) Curricular Development in Statistics Education. The Netherlands: International Statistical Institute and International Association for Statistical Education, 2005.

PALLAUTA, J. D.; ARTEAGA, P. Análisis de la complejidad semiótica de gráficos y tablas estadísticas. In: CAMPOS, C. R.; PAVAN, A. (Eds.). Investigações hispanobrasileiras em Educação Estatística. Brasil: Editora Akademy, 2020. p. 196-201.

PEEBLES, D.; ALI, N. Differences in comprehensibility between three-variable bar and line graphs. In: THE THIRTY-FIRST ANNUAL CONFERENCE OF THE COGNITIVE SCIENCE SOCIETY. Proceedings of the thirty-first annual conference of the Cognitive Science Society. Amsterdam, Netherlands: Cognitive Science Society, 2009. Disponível em: < http://csjarchive.cogsci.rpi.edu/Proceedings/2009/index.html $>$. Acesso em: 13 out. 2020

PÉREZ-ECHEVERRÍA, M. DEL P.; POSTIGO, Y.; MARÍN, C. Understanding of graphs in social science undergraduate students: selection and interpretation of graphs. Irish Educational Studies, v. 37, n. 1, p. 89-111, 2 jan. 2018.

PONTE, J. P. DA; FONSECA, H. Orientações curriculares para o ensino da Estatística. Análise comparativa de três países. Quadrante, v. 10, n. 1, p. 93-132, 30 jun. 2001.

RUZ, F.; MOLINA-PORTILLO, E.; MARTÍNEZ, F.; PEÑA, L.; CONTRERAS, J. M. Evaluación de la alfabetización estadística gráfica en futuros maestros de Educación Primaria. In: XVII CONGRESO DE ENSEÑANZA Y APRENDIZAJE DE LAS MATEMÁTICAS (CEAM XVII) (F. España, Ed.). Actas del XVII Congreso de Enseñanza y Aprendizaje de las Matemáticas: Matemáticas en tierra de cine. Almería: Sociedad Andaluza de Educación Matemáticas Thales, 2018

SCHONLAU, M.; PETERS, E. Graph Comprehension: An Experiment in Displaying Data as Bar Charts, Pie Charts and Tables With and Without the Gratuitous 3rd Dimension. Rochester, NY: Social Science Research Network, 23 set. 2008. Disponível em: <https://papers.ssrn.com/abstract=1272627>. Acesso em: 12 set. 2020.

SHARMA, S. Assessing Students' Understandıng of Tables and Graphs: Implications for Teaching and Research. International Journal of Educational Research and Technology, v. 4, p. 51-70, 2013.

SHAUGHNESSY, J. M.; GARFIELD, J.; GREER, B. Data Handling. In: BISHOP, A. J. et al. (Eds.). International Handbook of Mathematics Education: Part 1. Kluwer International Handbooks of Education. Dordrecht: Springer Netherlands, 1996. p. 205237.

WATSON, J.; FITZALLEN, N. The Development of Graph Understanding in the Mathematics Curriculum. Report for the NSW Department of Education and Training. State of New South Wales through the NSW, Department of Education and 
Training, 2010

WONG, D. M. The Wall Street Journal Guide to Information Graphics: The Dos and Don'ts of Presenting Data, Facts, and Figures. [s.1.] W.W. Norton \& Company, 2010.

WU, Y.; WONG, K. Y. Understanding of Statistical Graphs among Singapore Secondary Students. In: Mathematics Education. Series on Mathematics Education. [s.1.] World Scientific, 2009. v. 2p. 227-243.

ZIEFFLER, A.; GARFIELD, J.; FRY, E. What Is Statistics Education? In: BEN-ZVI, D.; MAKAR, K.; GARFIELD, J. (Eds.). International Handbook of Research in Statistics Education. Springer International Handbooks of Education. Cham: Springer International Publishing, 2018. p. 37-70.

Submetido em 15 de outubro de 2020. Aprovado em 01 de maio de 2021. 